\title{
The Fluoretec system for rapid diagnosis of bacteroides infections by direct immunofluorescence of clinical specimens
}

\author{
MARY PE SLACK, DT GRIFFITHS, HH JOHNSTON \\ From the Department of Bacteriology and Regional Public Health Laboratory, John Radcliffe Hospital, \\ Oxford
}

SUMMARY Fluoretec, a commercial kit for the rapid diagnosis by immunofluorescence of infections caused by Bacteroides spp was compared with a standard culture method. A total of 1010 specimens were tested for the presence of $B$ fragilis group by Fluoretec $\mathrm{F}$ and $\boldsymbol{B}$ melaninogenicus-oralis and asaccharolytic groups by Fluoretec $M$. Fluoretec $F$ was positive in $123 / 152$ specimens culturing $B$ fragilis group strains. Seventeen specimens were positive by Fluoretec $F$ but negative on culture. Fluoretec $\mathrm{M}$ was positive in 21 of 22 specimens from which $B$ melaninogenicus was cultured. The Fluoretec system was convenient in use, results being obtained within one hour of receipt of the specimen.

The use of immunofluorescence for the identification of Bacteroides spp was first described by Griffin $^{1}$ and further developed by Lombard and Dowell ${ }^{2}$ and by Stauffer et al. ${ }^{3}$ More recently a commercial kit, Fluoretec, has been produced (Pfizer Diagnostics).

Bacteria of the Bacteroides spp are important anaerobic pathogens in many clinical conditions. ${ }^{4}$ Bacteroides fragilis is predominant, accounting for more than $50 \%$ of bowel-associated anaerobic infections ${ }^{5}$ and $70 \%$ of Bacteroides spp isolated from the blood stream. ${ }^{6}$ Bacteroides spp of the melaninogenicus-oralis group are associated with infections of the female genital tract and mouth. ${ }^{5}$ The cultural requirements of anaerobes are more stringent and time-consuming than those of many organisms, causing an inevitable delay in the results. Early detection of an anaerobic infection ensures the rapid institution of effective chemotherapy.

Fluoretec (Pfizer Diagnostics) is a commercial kit containing polyvalent rabbit antisera to the $B$ fragilis group (Fluoretec $\mathrm{F}$ ) and to the $B$ melaninogenicusoralis group and asaccharolytic groups (Fluoretec M). These antisera are conjugated with fluorescein isothiocyanate to permit direct immunofluorescence of clinical specimens. Fluoretec $F$ contains antibodies to $B$ fragilis, $B$ vulgatus, $B$ distasonis, $B$ ovatus, and $B$ thetaiotaomicron. Fluoretec $\mathbf{M}$ contains antibodies to $B$ melaninogenicus, $B$ intermedius and $B$ asaccharolyticus. For brevity these will subsequently be

Accepted for publication 11 May 1981 referred to as $B$ fragilis group and $B$ melaninogenicus group.

The kit contains formalin-fixed positive controls of the $B$ fragilis group and the $B$ melaninogenicus group. It also includes a Rhodamine-B conjugated human and rabbit gamma globulin (Prestain). This is designed to react with organisms containing protein A, principally Staphylococcus aureus, thus blocking the non-specific reaction of protein $A$ with the Fc portion of the fluorescein conjugated anti-bacteroides antisera. Glass microscope slides with two ground glass circles are provided in the package.

In this study the reliability and ease of use of Fluoretec were evaluated. Results obtained by immunofluorescence were compared with the results of standard laboratory cultures.

\section{Material and methods}

\section{SPECIMENS}

A total of 1010 clinical specimens, all received in the routine diagnostic laboratory were tested for the presence of Bacteroides spp. Specimens were selected from the following clinical conditions:

Abdominal and genitourinary surgery, lung abscesses, cerebral abscesses, chronic soft tissue infections, otolaryngological and dental sepsis.

Each specimen was tested immediately by the Fluoretec method and was also examined by conventional culture techniques as described below. 
FLUORETEC

Upon receipt in the laboratory, thin smears of each specimen were made in the wells of a double well glass slide. Duplicate smears were prepared and stored. The smears were dried in air and then gently heat-fixed. A drop of Prestain was applied to each well and the slides were incubated for $15 \mathrm{~min}$ at room temperature in a moist chamber. The Prestain was removed by gentle blotting with blotting paper. A $20 \mu$ l volume of the $B$ fragilis group conjugate was applied to the first well and $20 \mu \mathrm{l}$ of $B$ melaninogenicus group conjugate was placed in the second well. The slide was incubated for a further $15 \mathrm{~min}$ at room temperature. After rinsing in distilled water for 15-20 s, the films were air dried. A large cover slip was placed over the smears and mounted in $90 \%$ buffered glycerol. The slides were examined at a magnification of $\times 63$ with a Leitz Dialux fluorescence microscope. Incident-light fluorescence with a $50 \mathrm{~W}$ ultra-high pressure mercury lamp and FITC interference filter was used. Results were recorded on a scale of $0,+,++$, and +++ where +++ represented numerous fluorescing bacteria and + represented scanty fluorescence. The fluorescence should be a bright apple-green outlining the organism. $B$ fragilis group is bacillary, $B$ melaninogenicus group is coccoid.

\section{B A CTERIOLOGY}

A smear of the specimen was prepared and stained by Gram's method. The number and nature of organisms present were noted. The specimens were plated on to two blood agar plates, $2 \%(\mu \mathrm{g} / \mathrm{ml})$ neomycin blood agar, McConkey's agar and inoculated into a cooked meat medium. In addition each specimen was plated on to prereduced Schaedler's medium (Oxoid) and Schaedler's medium with $2 \%(\mu \mathrm{g} / \mathrm{ml})$ neomycin. One blood agar, the neomycin blood agar and the two Schaedler's plates were placed in an anaerobic jar (Whitley 10 plate anaerobic jar) fitted with a $4 \mathrm{~g}$ cold catalyst sachet. The jar was evacuated to $760 \mathrm{~mm} \mathrm{Hg}$ and filled with a gas mixture of $10 \%$ hydrogen, $10 \%$ carbon dioxide and $80 \%$ nitrogen. A test for a secondary vacuum of $20 \mathrm{~mm} \mathrm{Hg}$ was performed after $10 \mathrm{~min}$.

Incubation of aerobic cultures was for $48 \mathrm{~h}$. Anaerobic cultures were inspected after $48 \mathrm{~h}$ incubation. The cooked meat medium was subcultured after $48 \mathrm{~h}$ incubation on to 2 blood agar plates, one of which was incubated aerobically, and the other was incubated anaerobically for a further 48 h. Anaerobic Gram-negative bacilli were assigned to the genus Bacteroides by their antibiotic disc resistance patterns ${ }^{7}$ using the Mastring identification test (Mast Laboratories, Liverpool).

Bacteroides strains were identified to the species level using API 20-A strips (API Laboratory Products, Farnborough, Hants).

If a specimen was positive by fluorescence but no 으 Bacteroides spp were isolated, all organisms that were isolated were tested for false-positive fluor- $\stackrel{\oplus}{\rightarrow}$ escence. Conversely if a Bacteroides sp was isolated but no fluorescence had been noted in the original specimen, the duplicate smear was stained and examined.

ANALYSIS OF RESULTS

Results for the two reagents, Fluoretec $F$ and $?$ Fluoretec M, were analysed separately. Only those $\vec{\omega}$ species of bacteroides included in the Fluoretec reagents have been included in the analysis. Sensi- $\frac{0}{0}$ tivity and specificity of the Fluoretec method was $\underset{+}{\infty}$ measured by comparison with the completed cultural results. Thus culture was deemed to give the $N$ "correct" result. No allowance was made for possible defects in the cultural system.

\section{Results}

A total of 1010 clinical specimens were examined between December 1977 and August 1979, $755 \stackrel{8}{3}$ specimens were received as dry swabs, 253 specimens were pus and the nature of two specimens was not $\overrightarrow{0}$ recorded. The anatomical sites from which to specimens were collected are shown in Table 1.

\section{Fluoretec $F$}

Fluoretec $F$ was positive in 123 of the 152 specimens in which $B$ fragilis group strains were detected by culture (Table 2). Cultural isolates from specimens $\unrhd$ recorded as negative by Fluoretec included $21 \overrightarrow{\overrightarrow{0}}$ strains of $B$ fragilis, 6 strains of $B$ vulgatus and 23

Table 1 Anatomical sources of 1010 clinical specimens

\begin{tabular}{lc}
\hline Source of specimen & No \\
\hline Gastrointestinal tract & 730 \\
Genitourinary tract & 188 \\
Thorax & 34 \\
Otolaryngology and dental & 35 \\
Cerebral abscess & 2 \\
Soft tissue infections & 21 \\
\hline
\end{tabular}

Table 2 Direct immunofluorescence using Fluoretec $F$ (B fragilis group)

\begin{tabular}{llr}
\hline Fluorescence & \multicolumn{2}{l}{ B fragilis group culture } \\
\cline { 2 - 3 } & Positive & Negative \\
\hline Positive & 123 & 17 \\
Negative & 29 & 841 \\
\hline
\end{tabular}


Table 3 Species of Bacteroides fragilis group obtained from 1010 clinical specimens

\begin{tabular}{lll}
\hline Species & \multicolumn{2}{l}{ Fluoretec $F$} \\
\cline { 2 - 3 } & $\begin{array}{l}\text { No of specimens } \\
\text { fluorescence }+v e\end{array}$ & $\begin{array}{l}\text { No of specimens } \\
\text { culture +ve }\end{array}$ \\
\hline B fragilis & 91 & 112 \\
B vulgatus & 20 & 26 \\
B distasonis & 4 & 6 \\
B ovatus & 1 & 1 \\
B thetaiotaomicron & 7 & 7 \\
& 123 & 152
\end{tabular}

One strain of $B$ fragilis and one strain of $B$ vulgatus were non-fluorescent.

Table 4 Direct immunofluorescence using Fluoretec $M$ (B melaninogenicus group)

\begin{tabular}{llc}
\hline Fluorescence & \multicolumn{3}{c}{ B melaninogenicus } & group culture \\
\cline { 2 - 3 } & Positive & Negative \\
\hline Positive & 21 & 21 \\
Negative & 1 & 967 \\
\hline
\end{tabular}

Table 5 Species of Bacteroides melaninogenicus group obtained from 1010 clinical specimens

\begin{tabular}{lll}
\hline Species & \multicolumn{2}{l}{ Fluoretec $M$} \\
\cline { 2 - 3 } & $\begin{array}{l}\text { No of specimens } \\
\text { fuorescence }+v e\end{array}$ & $\begin{array}{l}\text { No of specimens } \\
\text { culture }+v e\end{array}$ \\
\hline B melaninogenicus & 13 & 14 \\
B intermedius & 1 & 1 \\
B oralis & 1 & 1 \\
B asaccharolyticus & 6 & 6 \\
& 21 & 22 \\
\hline
\end{tabular}

strains of $B$ distasonis (Table 3). All but two of these strains (one $B$ fragilis and one $B$ vulgatus) gave positive results when tested with Fluoretec $F$.

There were 17 specimens positive by Fluoretec F but negative on culture. All isolates from these specimens were tested for non-specific fluorescence. Fluorescence was observed in organisms isolated from eight of the 17 specimens (5 Staph aureus, 3 Escherichia coli and 1 Streptococcus $\mathrm{sp}$ ).

Thus the sensitivity and specificity of Fluoretec $\mathrm{F}$ as judged by cultural results are $80.9 \%$ and $98 \%$ respectively.

\section{Fluoretec $M$}

Fluoretec $M$ gave positive results in 21 of the 22 specimens from which organisms of the $B$ melaninogenicus group were isolated (Tables 4 and 5). The isolate from the single specimen which was culturepositive but negative by Fluoretec $\mathbf{M}$ gave positive fluorescence on retesting with Fluoretec $M$. There were 21 specimens positive with Fluoretec $M$ from which no member of the $B$ melaninogenicus group was isolated. $B$ fragilis was isolated from four of these and a further eight gave growth of organisms which were shown to fluoresce non-specifically with Fluoretec M (3 Staph aureus, 1 E coli and 4 Strepto. coccus $\mathrm{spp}$ ). As judged by comparison with the cultural results Fluoretec $\mathbf{M}$ had a sensitivity of $95.5 \%$ and a specificity of $97.9 \%$.

\section{Effect of repeating Fluoretec tests}

When a Bacteroides sp was isolated, but no fluorescence had been observed in the original slide of the specimen the duplicate smear was stained and examined. In the case of repeat tests with Fluoretec $F$, nine extra specimens were found to be positive for $B$ fragilis. Repeat tests with Fluoretec $\mathbf{M}$ produced no extra positives. It must be emphasised that in order to obviate any tendency toward possible observer bias these repeat results do not form part of the analysis described above.

\section{Discussion}

The current popularity of gas liquid chromatography (GLC) for the rapid detection of infection by anaerobes testifies to the usefulness of such immediate techniques. However GLC requires the use of expensive equipment maintained by skilled staff. By contrast Fluoretec makes use of a technique which is already in operation in most microbiology laboratories. In our hands it proved convenient, results being obtainable in under an hour.

The evaluation of a new laboratory test is often complicated by the absence of a reliable external standard against which to measure its accuracy. Ideally a clinical test should predict the presence of a specific disease pattern in the patient. However assessments related to clinical measurement are, of course, impracticable in the short term. For this reason the researcher has to make do with comparisons with existing laboratory methodology while accepting that the so called standard is subject to considerable variation.

In our study we have compared Fluoretec with an anaerobic culture system which we believe to be the best available to the average diagnostic laboratory. In 112 specimens where culture was positive for Bacteroides fragilis 21 were negative by Fluoretec. Since 20 of these isolates were positive by fluorescence it may be assumed that these specimens contained too few organisms to be detected by Fluoretec. In this context it is of interest to note that while $30 \%$ of pus samples were positive by Fluoretec only $15 \%$ of dry swabs gave this result.

Some care is necessary in the interpretation of the fluorescence microscopy. Positive organisms should 
have a clear fluorescent outline and morphological similarity to the control organism. A number of organisms, notably Staph aureus, gave positive fluorescence despite the use of Rhodamine B conjugate and human gamma globulin (Prestain).

In a similar study Kaspar et al. ${ }^{8}$ described the use of indirect immunofluorescence to detect Bacteroides spp in clinical material. A $B$ fragilis capsular antiserum was more sensitive than Fluoretec detecting all $12 B$ fragilis strains with a $90.3 \%$ specificity. $B$ fragilis is the only species of the genus bacteroides to possess a polysaccharide capsule. ${ }^{9}$ A pooled whole organism antiserum to the $B$ fragilis group was more sensitive $(100 \%)$ than Fluoretec $F(80.9 \%)$ but far less specific $(64.3 \%)$ than Fluoretec F $(98 \%)$. They did not look for organisms of the $B$ melaninogenicus group.

Despite its obvious limitations we feel that the present study provides evidence of the effectiveness of the Fluoretec system. The concept of immediate detection and speciation of bacterial pathogens is an attractive one but the specific immunofluorescence technique, useful though it is in virology, has so far had very limited success in bacteriology. Fluoretec may well represent an advance in this field. Sensitivity of the test is good by bacteriological standards, particularly in view of the fact that no preincubation is needed. The numbers of false-positives are acceptably low. The reagents cover the species of bacteroides of greatest clinical interest ${ }^{5}$ with the exception of $B$ corrodens. Fluoretec is therefore a useful addition to the tests available for the detection of infections caused by Bacteroides spp particularly for laboratories not possessing equipment for gasliquid chromatography.
Fluoretec kits are available from Laboratory Impex Limited, Lion Road, Twickenham, Middlesex.

We are grateful to Dr Wayne W Laslie of Pfizer Diagnostics for supplying the Fluoretec kits and for his advice. We thank Dr RT Mayon-White for his assistance with the data analysis.

\section{References}

${ }^{1}$ Griffin Mary H. Fluorescent antibody techniques in the identification of Gram-negative non-spore forming anaerobes. Health Lab Sci 1970;7:78-83.

${ }^{2}$ Lombard GL, Dowell VR. Preparation of fluorescent antibody reagents for the identification of Bacteroides. Abstr Ann Meeting Amer Soc Microbiol 1972;93:95.

${ }^{3}$ Stauffer LR, Hill EO, Holland JW, Altemeir WA. Indirect fluorescent antibody procedure for the rapid detection and identification of Bacteroides and Fusobacterium in clinical specimens. J Clin Microbiol 1975;2:337-44.

${ }^{4}$ Finegold SM, Shepherd WE, Spaulding EH. Practical anaerobic bacteriology (Cumitech 5). Washington DC: American Society for Microbiology, 1977.

${ }^{5}$ Duerden BI. The identification of Gram-negative anaerobic bacilli isolated from clinical infections. $J \mathrm{Hyg}$ (Camb) 1980;84:301-13.

${ }^{6}$ Polk BF, Kaspar DL. Bacteroides fragilis. Subspecies in clinical isolates. Ann Intern Med 1977;86:569-71.

${ }^{7}$ Sutter VL, Finegold SM. Antibiotic disc susceptibility tests for rapid presumptive identification of Gramnegative anaerobic bacilli. Appl Microbiol 1971;21:13-20.

${ }^{8}$ Kaspar DL, Fiddian AP, Tabaqchali S. Rapid diagnosis o bacteroides infections by indirect immunofluorescenes assay of clinical specimens. Lancet $1979 ; \mathrm{i}: 239-42$.

${ }^{9}$ Kaspar DL. The polysaccharide capsule of Bacteroide fragilis subspecies fragilis: morphological and immuno.chemical definition. J Infect Dis 1976;133:79-87.

Requests for reprints to: Dr Mary PE Slack, Department of Bacteriology, Level 7, John Radcliffe Hospital, Headington, Oxford OX3 9DU, England. 